Publisher: Research Council of Science and Technology, Biratnagar, Nepal p. 89

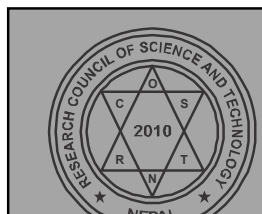

\title{
BIBECHANA
}

A Multidisciplinary Journal of Science, Technology and Mathematics

ISSN 2091-0762 (print), 2382-5340 (online)

Journal homepage: http://nepjol.info/index.php/BIBECHANA

\section{Significance of neutrino-neutrino interaction in neutrino oscillation in core-collapse supernova}

\author{
Prabandha Nakarmi ${ }^{1^{*}}$, Jeevan Jyoti Nakarmi ${ }^{\text {***}}$ \\ ${ }^{1}$ Department of Physics, Patan Multiple Campus, PatanDhoka, Nepal \\ ${ }^{2}$ Central Department of Physics, Tribhuvan University, Kirtipur, Nepal \\ *E-mail: nakarmipra@gmail.com \\ ${ }^{* *}$ E-mail: nakarmiji@tu.cdp.edu.np \\ Accepted for publication: December 19, 2014
}

\begin{abstract}
We study the possibility of neutrino-neutrino interaction inside the neutrino core of supernova $\left(\rho \geq 10^{10} \mathrm{~g} / \mathrm{cc}\right.$ ) and outside the neutrinosphere. The angular dependence of the neutrino-neutrino interaction Hamiltonian causes multi-angle effects that can lead either to oscillation or free streaming of neutrinos. If the angle between interactions is $\pi / 2$, the neutrinos are trapped inside neutrino core and chances of oscillation increases due to interaction. As the angle gradually changes, the chance of oscillation decreases and free streaming of neutrino can be observed out of core of supernova as shock waves.

DOI: http://dx.doi.org/10.3126/bibechana.v12i0.11780

(C) 2014 RCOST: All rights reserved.
\end{abstract}

Keywords: Neutrino-neutrino interaction; supernova; Hamiltonian.

\section{Introduction}

Since the discovery of neutrinos, there have been series of tremendous successful works carried out in the study of neutrinos. Neutrinos are very elusive in nature. Because of this nature, neutrinos rarely interact with matter and therefore very difficult to detect. The interaction of neutrino with matter is studied with great interest and the effect is known as MSW effect [1, 2]. MSW effect is mostly popular in solution of solar neutrino problem. Since the discovery of MSW effect, the study of neutrino oscillation has been accelerated.

After the Supernova explosion in 1987, the detail study of neutrino oscillation in core collapse supernova revealed surprising facts of neutrino oscillation. The huge amount of neutrinos produced in SN 1987A showed that the neutrinos gets trapped inside the core of supernova. Hence the study of neutrino-neutrino interaction must be accounted in the region where density of neutrino is very high such as core of supernova $\left(\rho \geq 10^{10} \mathrm{~g} / c c\right)$. Several works have investigated the conditions for the $v-v$ effects to be triggered. Neutrino-neutrino interaction may be one of the strong causes of neutrino oscillations. The no. of neutrinos formed during r-process nucleo-synthesis in core of supernova is very high; hence the probability of $v-v$ effects inside core increases.

The interaction of neutrino with other leptons and with neutrino itself is studied in a series of papers [1-7]. Also the neutrino oscillation in dense neutrino gases such as core of supernova is studied in paper [8], which showed the self-induced MSW effect. Also paper [9] shows that the angular 
Nakarmi and Nakarmi/ BIBECHANA 12 (2015) 89-95: p. 90

dependence of the neutrino-neutrino interaction potential causes multi-angle effects that can lead either to complete kinematical decoherence in flavor space or only to small differences between different trajectories. This nonlinear system switches abruptly between "self-maintained coherence" and "self-induced decoherence" among the angular modes, depending on the strength of the deleptonization flux.

The present work is to study about the multi-angle effects of $v-v$ interaction in core collapse supernova. The variation of interaction length due to angle variation is estimated through the angle dependent term of the Hamiltonian of $v-v$ interaction.

\section{Formalism}

The $v-v$ interaction Hamiltonian can be written as [10],

$H_{v v}=$

$\frac{G_{F}}{\sqrt{2} V} \int d^{3} p d^{3} q R_{p q}\left[a_{e}^{\dagger}(p) a_{e}(p) a_{e}^{\dagger}(q) a_{e}(q)+a_{x}^{\dagger}(p) a_{x}(p) a_{x}^{\dagger}(q) a_{x}(q)+a_{x}^{\dagger}(p) a_{e}(p) a_{e}^{\dagger}(q) a_{x}(q)+\right.$

$\left.a_{e}^{\dagger}(p) a_{x}(p) a_{x}^{\dagger}(q) a_{e}(q)\right]$

where, we defined

$$
R_{p q}=\left(1-\cos \vartheta_{p q}\right)
$$

$a_{e}^{\dagger}(p) \operatorname{and} a_{x}^{\dagger}(p)$ are the creation operators for the left-handed $v_{e}$ and $v_{x}$ with momentum $p$, respectively, and $a_{e}(p)$ and $a_{x}(p)$ are the corresponding annihilation operators [10-12]. Here, $\vartheta_{p q}$ is the angle between the momentum directions of the neutrinos with momenta $p$ and $q$ and box quantization conditions for a box with volume $\mathrm{V}$ is used in above equation.

The interaction between $v-v$ can also be written in the form, [7]

$$
H_{v v}=\sqrt{2} G_{F} \sum_{\alpha} \int \rho_{v_{\underline{\alpha}}}\left(\mathbf{q}^{\prime}\right)\left(1-\widehat{\mathbf{q}} \cdot \widehat{\mathbf{q}}^{\prime}\right) d_{n_{v_{\underline{\alpha}}}} d q^{\prime}-\sqrt{2} G_{F} \sum_{\alpha} \int \bar{\rho}_{v_{\underline{\alpha}}}\left(\mathbf{q}^{\prime}\right)\left(1-\widehat{\mathbf{q}} \cdot \widehat{\mathbf{q}}^{\prime}\right) d_{n_{\bar{v}_{\underline{\alpha}}}} d q^{\prime}
$$

where $d_{n_{v_{\underline{\alpha}}}}\left(d_{n_{\bar{v}_{\underline{\alpha}}}}\right)$ are the differential neutrino (anti-neutrino) number densities and $\rho_{v_{\underline{\alpha}}}\left(\bar{\rho}_{v_{\underline{\alpha}}}\right)$ is the density matrix for neutrinos (anti-neutrinos), encoding neutrino flavor conversion, whose expression is, for neutrinos

$$
\rho_{v_{\underline{\alpha}}} \equiv\left(\begin{array}{ccc}
\left|v_{e}\right|^{2} & v_{e} v_{\mu}^{*} & v_{e} v_{\tau}^{*} \\
v_{e}^{*} v_{\mu} & \left|v_{\mu}\right|^{2} & v_{\mu} v_{\tau}^{*} \\
v_{e}^{*} v_{\tau} & v_{\mu}^{*} v_{\tau} & \left|v_{\tau}\right|^{2}
\end{array}\right)
$$

and similarly for $\bar{\rho}_{v_{\underline{\alpha}}}$ for anti-neutrinos. In above equation, the neutrino flavor might have changed is indicated with a flavor underlined $\left(v_{\underline{\alpha}}\right)$. In Eq. 3, q, q'are different momenta of neutrinos and antineutrinos, also $\widehat{\mathbf{q}}=\mathbf{q} /|\mathbf{q}|$. The fact that their flavor might change in the interaction is encoded in the neutrino density matrix. 


\section{Application to the neutrino-neutrino interaction}

Let us apply the formalism and the results of the previous section to the Hamiltonian Eq. (3) describing neutrino propagation in a core-collapse supernova with the neutrino-neutrino interaction contribution. Here we treat the neutrino-neutrino contribution in the single-angle approximation.

From now, the time ( $\mathrm{t}$ ) is replaced with distance( $\mathrm{x}$ ). If we calculate the derivative of $\mathrm{H}$ necessary to determine the diagonal and off-diagonal terms of the Hamiltonian in the 'matter' basis, we find two contribution,

$$
\dot{H}=\dot{H}_{m a t}+\dot{H}_{v v}
$$

For the single-angle approximation, $H_{v v}$ in Eq. 3 becomes, [7]

$$
H_{v v}^{\text {s.a. }}=F(x)^{\text {s.a. }} G(\rho)^{\text {s.a. }}
$$

with the geometrical factor,

$$
F(x)^{s . a .}=\frac{\sqrt{2} G_{F}}{2 \pi R_{v}^{2}} \frac{1}{2}\left[1-\sqrt{1-\left(\frac{R_{v}}{x}\right)^{2}}\right]^{2}
$$

where $R_{v}$ is the radius of the neutrinosphere. The non-linear contribution is given by,

$$
G(\rho)^{s . a .}=\sum_{\alpha} \int\left(\rho_{v_{\underline{\alpha}}}\left(q^{\prime}\right) L_{v_{\underline{\alpha}}}\left(q^{\prime}\right)-\bar{\rho}_{\bar{v}_{\underline{\alpha}}}\left(q^{\prime}\right) L_{\bar{v}_{\underline{\alpha}}}\left(q^{\prime}\right)\right) d q^{\prime}
$$

with $L_{v_{\underline{\alpha}}}$, the neutrino flux at the same location for a neutrino of a flavor $\alpha$. The corresponding derivative $\dot{H}_{v v}$ includes contributions from both the derivative of the geometrical factor and the density matrices, i.e.

$$
\dot{H}_{v v}^{s . a .}=\dot{F}(x)^{s . a \cdot} G(\rho)^{s . a .}+F(x)^{s . a \cdot} \dot{G}(\rho)^{\text {s.a. }}
$$

where the calculation of $\dot{F}(x)$ is straight forward and the non-linear term is obtained using of the Liouville Von-Neumann equation which gives,

$$
\dot{G}(\rho)^{s . a .}=-i \sum_{\alpha} \int\left(\left[H, \rho_{v_{\underline{\alpha}}}\left(q^{\prime}\right)\right] L_{v_{\underline{\alpha}}}\left(q^{\prime}\right)+\left[\bar{H}, \rho_{\bar{v}_{\underline{\alpha}}}\left(q^{\prime}\right)\right]^{*} L_{\bar{v}_{\underline{\alpha}}}\left(q^{\prime}\right)\right) d q^{\prime}
$$

where $\bar{H}$ indicates the Hamiltonian for anti-neutrinos. In the case of anti-neutrinos, Eq. (10) holds by replacing $H \leftrightarrow \bar{H}$ and $v \leftrightarrow \bar{v}$.

From the derived relations, we can identify two interesting conditions to be fulfilled:

(i) For the neutrino-neutrino interaction effects to occur

(ii) For the onset of bipolar oscillations.

\section{Multi-angle Approximation}

In the multi-angle approximation, assuming spherical geometry for the neutrino sphere, $H_{v v}$ of Eq. (3) reads as

$$
H_{v v}^{m . a .}=\frac{\sqrt{2} G_{F}}{2 \pi R_{v}^{2}} \sum_{\alpha} \int_{0}^{\infty} \int_{\cos \theta_{\max }}^{1} d q^{\prime} d \cos \theta^{\prime}\left(1-\cos \theta \cos \theta^{\prime}\right)
$$




$$
\left[\rho_{v_{\underline{\alpha}}}\left(q^{\prime}, \theta^{\prime}\right) L_{v_{\underline{\alpha}}}\left(q^{\prime}\right)-\rho_{\bar{v}_{\underline{\alpha}}}^{*}\left(q^{\prime}, \theta^{\prime}\right) L_{\bar{v}_{\underline{\alpha}}}\left(q^{\prime}\right)\right]
$$

with,

$$
\cos \theta_{\max }=\sqrt{1-\left(R_{v} / x\right)^{2}}
$$

Using Leibniz Integral Rule:

$\frac{\partial}{\partial z} \int_{a(z)}^{b(z)} f(y, z) d t=\int_{a(z)}^{b(z)} \frac{\partial f(y, z)}{\partial z} d t+f(b(z), Z) \frac{\partial b(z)}{\partial z}-f(a(z), z) \frac{\partial a(z)}{\partial z}$

the derivative for Eq. (11) is

$$
\dot{H}_{v v}^{m . a .}=\frac{\sqrt{2} G_{F}}{2 \pi R_{v}^{2}} \sum_{\alpha} \int_{0}^{\infty} d q^{\prime}\left(R\left(\theta^{\prime}, q^{\prime}\right)-S(x) T\left(q^{\prime}\right)\right)
$$

with,

$$
\begin{aligned}
& R\left(\theta^{\prime}, q^{\prime}\right)= \\
& \int_{\cos \theta_{\max }}^{1} d \cos \theta^{\prime}\left(-i\left(1-\cos \theta \cos \theta^{\prime}\right)\left(\left[H, \rho_{v_{\underline{\alpha}}}\left(q^{\prime}, \theta^{\prime}\right)\right] L_{v_{\underline{\alpha}}}\left(q^{\prime}\right)+\left[\bar{H}, \rho_{\bar{v}_{\underline{\alpha}}}\left(q^{\prime}, \theta^{\prime}\right)\right]^{*} L_{\bar{v}_{\underline{\alpha}}}\left(q^{\prime}\right)\right)\right) \\
& S(x)=\frac{\left(R_{v} / x\right)^{2}}{x \sqrt{1-\left(R_{v} / x\right)^{2}}} \\
& T\left(q^{\prime}\right)=\left(1-\cos \theta \cos \theta_{\max }\right)\left[\rho_{v_{\underline{\alpha}}}\left(q^{\prime}, \theta_{\max }\right) L_{{\underline{v_{\underline{\alpha}}}}_{1}}\left(q^{\prime}\right)-\rho_{\bar{v}_{\underline{\alpha}}}^{*}\left(q^{\prime}, \theta_{\max }\right) L_{\bar{v}_{\underline{\alpha}}}\left(q^{\prime}\right)\right]
\end{aligned}
$$

\section{Numerical Results and Discussion}

Here we present results of multi-angle variation in neutrino oscillation due to $v-v$ interaction and the probability of neutrino oscillation in core collapse supernova due to $v-v$ interaction by using Eq. (12), (16), and (18). In this calculation, spherical symmetry is assumed. The distance $(x)$ is taken in $\mathrm{Km}$ and the value ofcos $\theta_{\max }$ ranges between -1 to +1 . The radius of neutrino sphere $\left(R_{v}\right)$ is taken as $10 \mathrm{Km}$.

Figure 1 and 2 shows the variation of distance $x$ with interaction angle. This variation shows the $v$-oscillation probabilities, as a function of the distance in supernova. The graphs obtained clearly indicate strong $v-v$ interaction atcos $\theta_{\max }=0 \quad$ i.e. $\theta_{\max }=\pi / 2$ where the length of interaction is equals to $R_{v}$. The possibility of $v-v$ interaction in the range $x \rightarrow R_{v}$ shows the probability of $v$-oscillation in the dense core of SN. If the value of $\cos \theta_{\max }$ is altered, then we see the length of interaction increases exponentially, and hence the $v-$ oscillation due to $v-v$ interaction will be rare. Also when the angle of interaction is about $25^{\circ}$ or $155^{\circ}$, the graph indicates free streaming of neutrinos in the form of shock waves. Some of the recent study of core collapse supernovae justifies the above conclusion.

Substituting equation (12) in equation (16) and using simple algebra we obtain,

$$
S(x)=\frac{\sin ^{3} \theta_{\max }}{R_{\nu} \cos \theta_{\max }}
$$


When we plot $S(x)$ against $x$ and $\theta_{\max }$, figure 3 and 4 are obtained respectively. The graph thus obtained resembles with the result obtained from figure 1 and 2 . The value of $S(x)$ is also varied with varying length and angle.

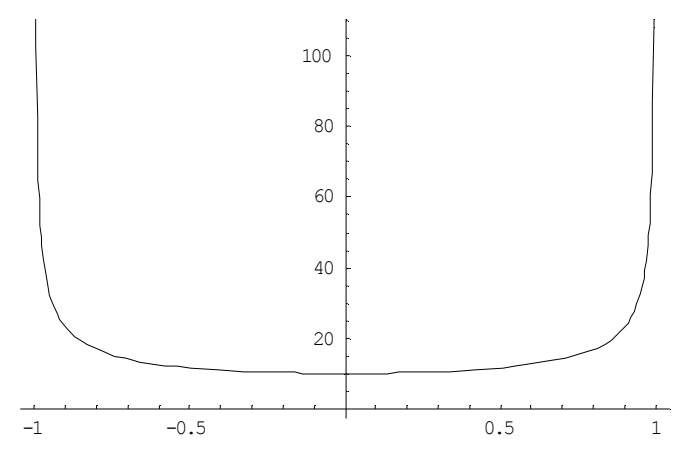

Fig. 1: Graph between $\cos \theta_{\max }$ along $\mathrm{x}$-axis and distance $\mathrm{x}(\mathrm{in} \mathrm{Km})$ along $\mathrm{y}$-axis.

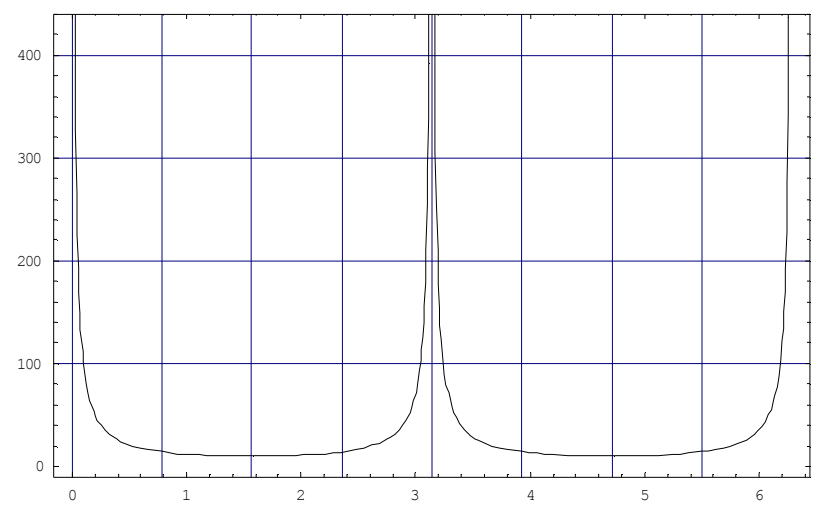

Fig.2: Graph between $\theta_{\max }($ ranges 0 to $2 \pi$ ) along $\mathrm{x}$-axis and $\mathrm{x}$ (in $\mathrm{Km}$ ) along $\mathrm{y}$-axis.

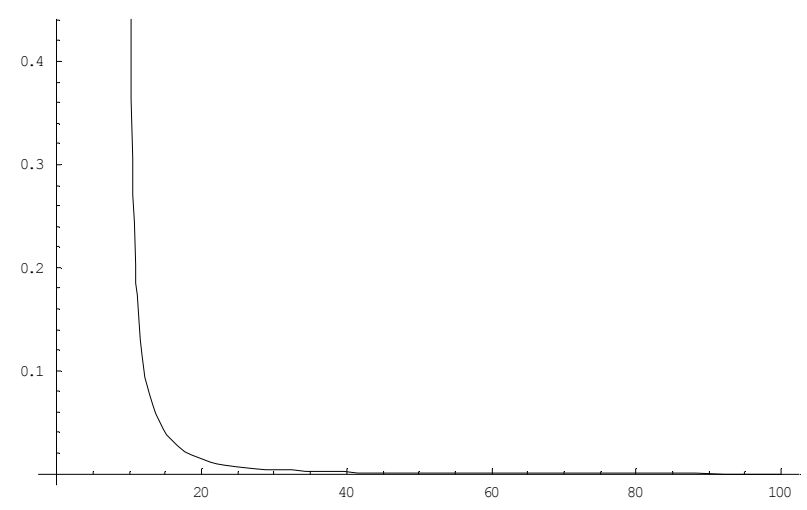

Fig. 3: Graph between $x($ in $K m)$ along $x$-axis and $S(x)$ along $y$-axis. 
Figure 3 shows that at $x \rightarrow R_{v}$, neutrinos are trapped due to interaction and the possibility of $v$-oscillation is high at core of SN. Also Figure 4 supports the idea of multi-angle effects in $v$-oscillation due to interaction. As the angle is $\pi / 2$, the probability of $v$-oscillation is maximum, and outside the neutrino core, probability falls exponentially.

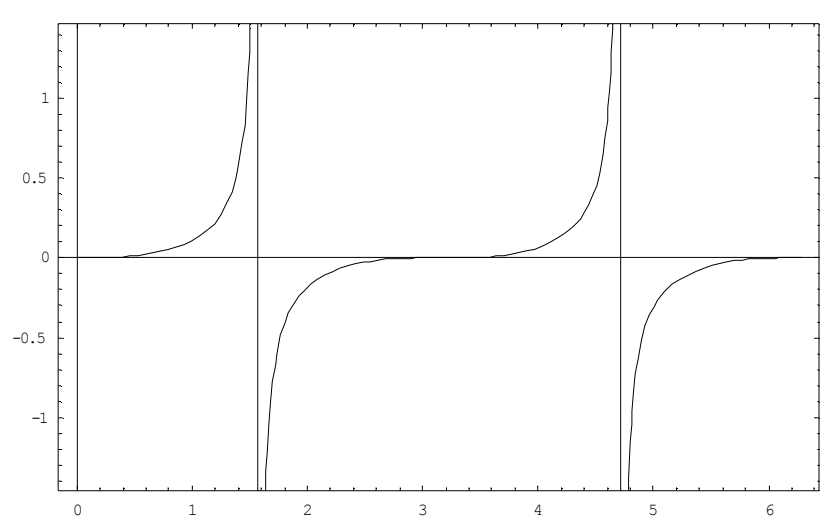

Fig. 4: Graph between $\theta_{\max }$ along $x$-axis and $S(x)$ along $y$-axis.

\section{Conclusion}

A neutrino gas is a highly nonlinear system in which unexpected phenomenon may occur. In core collapse supernova we have observed two types of behavior. There is a self-induced MSW effect due to neutrino-neutrino interaction inside the core of supernova $\left(\rho \geq 10^{10} \mathrm{~g} / \mathrm{cc}\right)$ and free streaming of neutrinos outside core of supernova in the form of shock waves. The matter induced mixing angle evolved from a value around $\pi / 2$ to a value near zero. We found that neutrino-neutrino interactions caused the oscillations to remain in phase and decoherence did not occur. This phenomenon took place for a relatively dense system and arouse due to the nonlinear nature of the problem.

\section{References}

[1] L. Wolfenstein, Phys. Rev. D 17 (1978) 2369; 20 (1979) 2634. http://dx.doi.org/10.1103/PhysRevD.45.1782

[2] S. P. Mikheev and A. Yu. Smirnov, Yad. Fiz. 42, (1985) 1441.

[3] G. Raffelt, G. Sigl and L. Stodolsky Phys. Rev. D 45 (1992) 1782.

[4] A. B. Balantekin and G. M. Fuller, Phys. Lett. B 471 (1999) 195

[5] S. P. Mikheev and A. Y. Smirnov, Nuovo Cimento C (1976) 917.

[6] A. B. Balantekin and G. M. Fuller, J. Phys. G: Nucl. Part. Phys. 29 (2003) 2513.

[7] Sébastien Galais, James Keneller, and Cristina Volpe [astro-ph.SR] 26 Aug, 2011. 
Nakarmi and Nakarmi/ BIBECHANA 12 (2015) 89-95: p. 95

[8] Stuart Samuel, Phys. Rev. D, 48 (1993) 1462-1477.

http://dx.doi.org/10.1103/PhysRevD.48.1462

[9] A. Esteban-Pertel, S Pastor, R. Tomas, G. G Raffelt, G. Sigl, Journal of Phys. Conference Series 120 (2008) 052021.

[10] A.B. Balantekin and Y. Pehlivan, J. Phys. G: Nucl. Part. Phys., 34 (2007) 47-65. http://dx.doi.org/10.1088/0954-3899/34/3/N01

[11] G. Raffelt, G. Sigl and L. Stodolsky, Phys. Rev. D 45 (1992) 1782. http://dx.doi.org/10.1103/PhysRevD.45.1782

[12] A. B. Balantekin and G.M. Fuller, Phys. Lett. B, 471 (195). 